\title{
BMJ Open Hokuriku-plus familial hypercholesterolaemia registry study: rationale and study design
}

Hayato Tada (D) , ${ }^{1}$ Hirofumi Okada, ${ }^{1}$ Shohei Yoshida, ${ }^{1}$ Masaya Shimojima, ${ }^{1}$ Akihiro Nomura (D) , ${ }^{1}$ Toyonobu Tsuda, ${ }^{1}$ Mika Mori, ${ }^{1}$ Shin-ichiro Takashima, ${ }^{1}$ Takeshi Kato, ${ }^{1}$ Soichiro Usui, ${ }^{1}$ Kenji Sakata, ${ }^{1}$ Kenshi Hayashi, ${ }^{1}$ Noboru Fujino, ${ }^{1}$ Akihiro Inazu, ${ }^{2}$ Shizuko Takahara, ${ }^{3}$ Yasuhito Imai, ${ }^{3}$ Takao Matsubara, ${ }^{4}$

Atsushi Nohara, ${ }^{4}$ Kenji Miwa, ${ }^{4}$ Masanobu Namura, ${ }^{5}$ Hidenobu Terai, ${ }^{5}$ Taiji Yoshida, ${ }^{5}$ Tsutomu Araki, ${ }^{6}$ Masahiro Minamoto, ${ }^{7}$ Toru Aburao, ${ }^{7}$ Yuji Ito, ${ }^{8}$ Chiaki Nakanishi, ${ }^{8}$ Suguru Kawasaki, ${ }^{9}$ Yasuhiro Todo, ${ }^{10}$ Junji Koizumi, ${ }^{11}$ Yoshihito Kita, ${ }^{12}$ Hiroshi Matsumoto, ${ }^{12}$ Hiroaki Shintaku, ${ }^{12}$ Akihiko Hodatsu, ${ }^{13}$ Hidekazu Ino, ${ }^{14}$ Toshinori Higashikata, ${ }^{15}$ Mutsuko Takata, ${ }^{15}$ Katsushi Misawa, ${ }^{16}$ Masato Yamaguchi, ${ }^{17}$ Yoshihiro Noji, ${ }^{17}$ Kazuo Osato, ${ }^{18}$ Tomohito Mabuchi, ${ }^{18}$ Taro Ichise, ${ }^{18}$ Bunji Kaku, ${ }^{19}$ Shoji Katsuda, ${ }^{19}$ Manabu Fujimoto, ${ }^{20}$ Katsuharu Uchiyama, ${ }^{20}$ Kensuke Fujioka, ${ }^{20}$ Takuya Nakahashi, ${ }^{21}$ Tsuyoshi Nozue, ${ }^{22}$ Ichiro Michishita, ${ }^{22}$ Kazuo Usuda, ${ }^{23}$ Kanichi Otowa, ${ }^{23}$ Kazuyasu Okeie, ${ }^{24}$ Satoshi Hirota, ${ }^{25}$ Isao Aburadani, ${ }^{25}$ Keisuke Kurokawa, ${ }^{26}$ Osamu Takatori, ${ }^{26}$ Shunichiro Hondo, ${ }^{27}$ Hiroyuki Oda, ${ }^{27}$ Shigeo Takata, ${ }^{28}$ Hisayoshi Murai, ${ }^{28}$ Masaki Kinoshita, ${ }^{29}$ Hideo Nagai, ${ }^{30}$ Yoshiteru Sekiguchi, ${ }^{30}$ Satoru Sakagami, ${ }^{31}$ Wataru Omi, ${ }^{31}$ Chikara Fujita, ${ }^{32}$ Tatsuo Katsuki, ${ }^{32}$ Hiroshi Ootsuji, ${ }^{33}$ Atsushi Igarashi, ${ }^{33}$ Manabu Nakano, ${ }^{34}$ Seiichiro Okura, ${ }^{35}$ Koji Maeno, ${ }^{35}$ Yasuhito Mitamura, ${ }^{36}$ Naoki Sugimoto, ${ }^{37}$ Masakazu Yamamoto, ${ }^{38}$ Hironobu Akao, ${ }^{39}$ Kouji Kajinami, ${ }^{39}$ Masayuki Takamura, ${ }^{1}$ Masa-aki Kawashiri ${ }^{1}$

To cite: Tada H, Okada $\mathrm{H}$, Yoshida S, et al. Hokuriku-plus familial hypercholesterolaemia registry study: rationale and study design. BMJ Open 2020;10:e038623. doi:10.1136/ bmjopen-2020-038623

\section{- Prepublication history for} this paper is available online. To view these files, please visit the journal online (http://dx.doi. org/10.1136/bmjopen-2020038623).

Received 19 March 2020 Revised 13 July 2020 Accepted 04 August 2020

Check for updates

(C) Author(s) (or their employer(s)) 2020. Re-use permitted under CC BY-NC. No commercial re-use. See rights and permissions. Published by BMJ.

For numbered affiliations see end of article.

Correspondence to

Hayato Tada;

ht240z@sa3.so-net.ne.jp

\section{ABSTRACT}

Introduction Familial hypercholesterolaemia (FH) is an autosomal-dominant inherited genetic disease. It carries an extremely high cardiovascular risk associated with significantly elevated low-density lipoprotein (LDL) cholesterol. The diagnostic rate of this disease in some European nations is quite high, due to the presence of multiple prospective registries. On the other hand, few data-and in particular multicentre data-exist regarding this issue among Japanese subjects. Therefore, this study intends to assemble a multicentre registry that aims to comprehensively assess cardiovascular risk among Japanese FH patients while taking into account their genetic backgrounds.

Methods and analysis The Hokuriku-plus FH registry is a prospective, observational, multicentre cohort study, enrolling consecutive FH patients who fulfil the clinical criteria of FH in Japan from 37 participating hospitals mostly in Hokuriku region of Japan from April 2020 to March 2024. A total of 1000 patients will be enrolled into the study, and we plan to follow-up participants over 5 years. We will collect clinical parameters, including lipids, physical findings, genetic backgrounds and clinical events covering atherosclerotic and other important events, such as malignancies. The primary endpoint of this study is new atherosclerotic cardiovascular disease (ASCVD) events. The secondary endpoints are as follows: LDL
Strengths and limitations of this study

This will be the first prospective multicentre cohort study in Japan to assess the risk of future atherosclerotic cardiovascular disease (ASCVD) and other clinical events among patients with familial hypercholesterolaemia (FH).

- We are performing comprehensive genetic testing for all of the participants. This will both enable us to confirm the diagnosis of FH and future ASCVD risk, and also facilitate decision-making over treatment as well as promote cascade screening among the patients' relatives. In addition, these data will help us improve the current clinical diagnostic criteria of FH in Japan, and potentially across the world.

- Although this study is conducted across a number of centres, it is not a nationwide study.

cholesterol, secondary ASCVD events and the occurrence of other diseases including hypertension, diabetes and malignancies.

Ethics and dissemination This study is being conducted in compliance with the Declaration of Helsinki, the Ethical Guidelines for Medical and Health Research Involving Human Subjects, and all other applicable laws and guidelines in Japan. This study protocol has been 
approved by the Institutional Review Board at Kanazawa University. We will disseminate the final results at international conferences and in a peer-reviewed journal.

Trial registration number UMIN000038210.

\section{INTRODUCTION}

Familial hypercholesterolaemia (FH) is an autosomaldominant inherited disorder. Patients with heterozygous FH account for approximately $0.5 \%-0.2 \%$ of the general population, equivalent to 1 in 200-500 individuals. ${ }^{1-5}$ Lowdensity lipoprotein $(\mathrm{LDL})$ receptor $(L D L R)$, proprotein convertase subtilisin/kexin type 9 (PCSK9), apolipoprotein B $(A P O B)$ and $L D L R$ adaptor protein 1 (LDLRAP1) are the major causative genes of FH. Since FH is characterised by hyper-LDL-cholesterolemia and systemic xanthomas from infancy, it is one of the leading causes of premature coronary artery disease. ${ }^{6}$ Some studies suggest that early diagnosis and appropriate LDL-lowering therapies could lead to a better prognosis for $\mathrm{FH}$ patients. ${ }^{7-9}$ In that sense, several registries of this disease have been organised to validate these important suggestions, such as the Simon Broome Familial Hyperlipidaemia Registry in the UK, ${ }^{10}$ CASCADE FH Registry in the USA ${ }^{11}$ and SAFEHEART registry in Spain. ${ }^{12}$

For more than 50 years, our study group has been helping to clarify genotype-phenotype associations among Japanese FH patients; ${ }^{6}$ however, few data-and in particular multicentre data-exist regarding this issue among Japanese subjects. On the other hand, we recently established a worthwhile scheme of comprehensive $\mathrm{FH}$ genetic testing using next-generation sequencing. ${ }^{13}$ Under these conditions, we intend to organise a multicentre registry that seeks to assess comprehensively atherosclerotic cardiovascular disease (ASCVD) risks among Japanese FH patients taking into account their genetic backgrounds. We believe that such information could lead to a better understanding of this important genetic disorder, both in Japanese patients, and also in patients elsewhere in the world.

\section{METHODS AND ANALYSIS Overall study design}

Recruitment for this prospective, observational, multicentre cohort study extends from April 2020 to March 2024. We are enrolling consecutive FH patients who fulfil the clinical criteria of the disorder in Japan from 37 participating hospitals located mostly in Hokuriku region (figure 1). Hokuriku, which comprises the prefectures of Toyama, Ishikawa and Fukui, is located in the central area of Japan's main island and is home to around 3 million people. It is of note that this region expects to see a relatively low population movement over the 5 years we have planned for follow-up, which in turn could lead to high proportion of subjects remaining in the study. A total of 1000 patients will be enrolled. Figure 1 shows the distribution of institutes that participated in this study and table 1 outlines the overall follow-up schedule. The primary

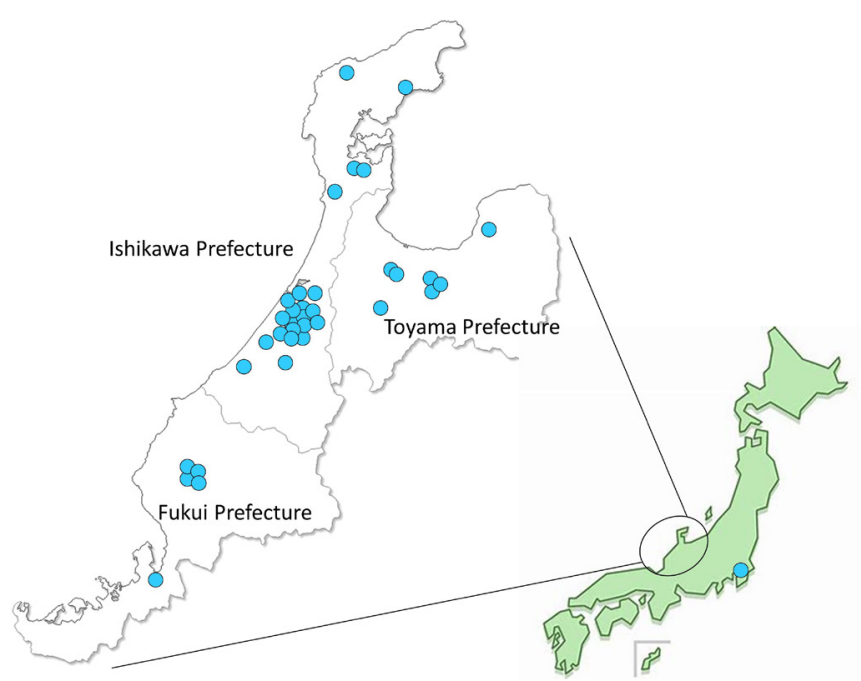

Figure 1 Participating study centres, including 37 hospitals across the Hokuriku region of Japan Blue circles indicate the hospitals participating in this study. Thirty-one of the 37 hospitals are located in the Hokuriku region of Japan and one hospital is located in the Tokyo area.

outcome of this study is the occurrence of new onset of ASCVD events. The secondary outcomes are as follows: LDL cholesterol, secondary ASCVD events and occurrence of other diseases such as hypertension, diabetes and malignancies.

Basic variables include date of birth, gender, height, bodyweight and waist circumference. Physical findings include cutaneous xanthomas, Achilles tendon thickness $(\geq 9.0 \mathrm{~mm}$ by X-ray) and arcus corneae. Family history includes the family history of premature coronary artery disease (male: $\leq 55$ year, female: $\leq 65$ year), or that of FH. Complications include hypertension, diabetes, any malignancies, ischaemic or haemorrhagic stroke or any unknown critical status. Genetic analyses include the presence and type of pathogenic mutation in $\mathrm{FH}$-associated genes. Prior ASCVD include carotid atherosclerosis ( $\geq 75 \%$ stenotic lesion), coronary atherosclerosis $(\geq 75 \%$ stenotic lesion), peripheral artery diseases $(\geq 75 \%$ stenotic lesion) or aortic valve stenosis (max. velocity $\geq 3 \mathrm{~m} / \mathrm{s}$ ). Laboratory data include blood counts, total cholesterol, triglycerides, HDL cholesterol, LDL cholesterol, lipoprotein (a), apolipoproteins, plasma glucose, HbAlc, aspartate aminotransferase, alanine transaminase, $\gamma$-glutamyl transpeptidase, alkaline phosphatase, lactate dehydrogenase, blood urea nitrogen, creatinine, uric acid, sodium, potassium, chloride, C-reactive protein and urinalysis. Each institution is responsible for maintaining the quality of its laboratory measurements. Events include new onset of ASCVD such as carotid atherosclerosis, coronary atherosclerosis, peripheral artery disease or aortic valve stenosis, complications including hypertension, diabetes, any malignancies, ischaemic or haemorrhagic stroke or any unknown critical status.

This study is being conducted in compliance with the Declaration of Helsinki, the Ethical Guidelines for Medical and Health Research Involving Human Subjects 
Table 1 Assessment and evaluation schedule of this study

\begin{tabular}{llllllll}
\hline & Baseline & 1 year & 2 year & 3year & 4 year & 5year & At events \\
\hline Basic variables & $\mathrm{X}$ & $\mathrm{X}$ & $\mathrm{X}$ & $\mathrm{X}$ & $\mathrm{X}$ & $\mathrm{X}$ & $\mathrm{X}$ \\
$\begin{array}{l}\text { Physical } \\
\text { findings }\end{array}$ & $\mathrm{X}$ & & & & & \\
Family history & $\mathrm{X}$ & & & & & \\
Smoking/alcohol & $\mathrm{X}$ & & & & & $\mathrm{X}$ \\
Medications & $\mathrm{X}$ & $\mathrm{X}$ & $\mathrm{X}$ & $\mathrm{X}$ & $\mathrm{X}$ & $\mathrm{X}$ & $\mathrm{X}$ \\
Complications & $\mathrm{X}$ & $\mathrm{X}$ & $\mathrm{X}$ & $\mathrm{X}$ & $\mathrm{X}$ & $\mathrm{X}$ & $\mathrm{X}$ \\
Genetic analyses & $\mathrm{X}$ & $\mathrm{X}$ & & & & $\mathrm{X}$ & $\mathrm{X}$ \\
Prior ASCVD & $\mathrm{X}$ & $\mathrm{X}$ & $\mathrm{X}$ & $\mathrm{X}$ & $\mathrm{X}$ & $\mathrm{X}$ & $\mathrm{X}$ \\
Laboratory data & $\mathrm{X}$ & $\mathrm{X}$ & $\mathrm{X}$ & $\mathrm{X}$ & $\mathrm{X}$ & $\mathrm{X}$ & $\mathrm{X}$ \\
Events & & & & & & $\rightarrow$ &
\end{tabular}

ASCVD, atherosclerotic cardiovascular disease.

and all other applicable laws and guidelines in Japan. In addition, this study protocol (V.1.0, dated 8 September 2019) has been approved by the Institutional Review Board at Kanazawa University (No. 2019-114).

\section{Study participants}

Patients diagnosed clinically with FH from April 2020 to March 2024 are being recruited, and we plan to follow-up on participants over 5 years. Of note, only participants fulfilling all inclusion criteria are being enrolled in this study (box 1) and those with either of the exclusion criteria are excluded from this trial (box 2). We are obtaining written informed consent from all participants using forms that have been approved by the IRB at Kanazawa University (see online supplementary material). Trial participants are required to understand the contents of the consent form before giving their acceptance; moreover, these forms must be dated and signed by both trial participants and investigators. On obtaining consent, the first copy of the consent form will be kept at the hospital and the other by the patient and will not be collected after the completion of this trial. Furthermore, all participants are informed that their medical care will not be affected if they refuse to enrol in the trial. They are free to withdraw their consent at any time during the study period, at their own discretion. To protect privacy, all the information will be anonymised at each institution before data are entered.

\section{Box 1 Inclusion criteria}

Patients with both of the following criteria are included.

1. Diagnosed with familial hypercholesterolaemia, according to criteria set by the Japan Atherosclerosis Society.

2. Willingness to provide written informed consent.

\section{Patient and public involvement}

This research is being carried out without patient involvement. Patients are not being invited to comment on the study design nor are they being consulted in developing patient-relevant outcomes or interpreting the results. Patients were not invited to contribute to the writing or editing of this document for readability or accuracy.

\section{Genetic testing}

We intend to sequence exons of four FH-related genes (LDLR, PCSK9, APOB and LDLRAP1) as well as other genes associated with Mendelian lipid disorders, such as ATP-binding cassette subfamily $\mathrm{G}$ member 5 (ABCG5) and ATP-binding cassette subfamily $\mathrm{G}$ member 8 (ABCG8) using the Illumina MiSeq system. The variant is defined as causal when it fulfils either of the following criteria: (a) it is registered as pathogenic/likely pathogenic in the ClinVar database; (b) minor allele frequency is $<1 \%$ in the East Asian population with (i) protein-truncating variants (non-sense, canonical splice sites or frameshift) and (ii) missense variants in the $L D L R$ gene that five in silico damaging scores (SIFT, PolyPhen-2 HDIV, PolyPhen-2 HVAR, MutationTaster2 and LRT) all predicted as pathogenic; (c) missense variants reported as pathogenic in the Japanese population (PCSK9 p.Val4Ile and p.Glu32Lys) and (d) eXome-Hidden Markov Model software predicted as copy number variations in the $L D L R$ gene (large duplication/large deletion). Details are described elsewhere. ${ }^{13}$

\section{Box 2 Exclusion criteria}

Familial hypercholesterolaemia patients with both of the following criteria are excluded.

1. Considered inappropriate to participate by their doctors in charge. 


\section{Data collection}

Attending physicians and research assistants are collecting data under the supervision of the clinicians responsible for this study at each participating hospital. The primary source of the information obtained is through electronic health records at each institute. Data are recorded through electronic data capture (EDC) using the browserbased, metadata-driven EDC software REDCap. ${ }^{14}$ The proposed time frame for data collection after the initial enrolment is shown in table 1.

\section{Outcomes}

The primary endpoint of this study is new ASCVD events. The secondary endpoints are as follows: LDL cholesterol, secondary ASCVD events and the occurrence of other diseases such as hypertension, diabetes or malignancies. We defined ASCVD events as fatal or non-fatal myocardial infarction; angina pectoris; ischaemic diseases including carotid atherosclerosis ( $\geq 75 \%$ stenotic lesion), coronary atherosclerosis ( $\geq 75 \%$ stenotic lesion), peripheral artery disease $(\geq 75 \%$ stenotic lesion) or aortic valve stenosis (max velocity $\geq 3 \mathrm{~m} / \mathrm{s}$ ) and ischaemic or haemorrhagic stroke.

\section{Data collection schedule}

Table 1 lists the overall data collection schedule for this study. Follow-up visits will be conducted in outpatient clinics at Kanazawa University Hospital and its affiliated institutions across the Hokuriku region of Japan. Follow-up interval, as well as the variables that are measured at certain points, will be decided according to the clinical guideline for $\mathrm{FH}^{1}$ and the patient's condition. Nevertheless, the clinical variables listed above will be collected at least once a year during the follow-up period and collected centrally through the EDC system. We will collect data at any point when an event occurs.

Data monitoring is conducted by an independent member of staff. The trial institution is monitored after the initial enrolment of patients and subsequently every 6 months until the case report form of the last participant has been obtained. The trial database is also monitored and reviewed by the independent staff member, and data queries will be raised if necessary.

\section{Statistical analysis}

The baseline profiles are described by the mean and $\mathrm{SD}$, median and quantiles (continuous variables) or proportion (categorical variables). A multivariate Cox proportional-hazards model will be used to evaluate the factors associated with new ASCVD events. In addition, this model will also be used to evaluate factors associated with LDL cholesterol, secondary ASCVD events and other complications, accounting for variables including age, gender, body mass index (baseline or its change), waist circumference (baseline or its change), hypertension, diabetes and smoking. We will compare the variables between patients with and without events using the t-test, Mann-Whitney U-test, Fisher's exact test or linear or logistic regression adjusted by appropriate covariates stated above. Finally, $\mathrm{p}<0.05$ will be considered statistically significant for the primary endpoint. Statistical analysis will be performed using SAS V.9.3 or R software V.3.4.1 or above.

\section{Patient and public involvement}

Patients and the public are not being involved in the development of the research questions and outcome measures, the study's design, the assessment of interventions in the study or recruitment and conduct. We will disseminate the final results to the study participants after these are published in a peer-reviewed journal.

\section{DISCUSSION}

The Hokuriku-plus FH registry is a prospective, observational, multicentre cohort study designed to capture recent trends in clinical characteristics, genetic backgrounds, management and prognosis of patients with FH in real-world clinical practice of Japan. The registry includes all patients admitted to participating centres with $\mathrm{FH}$, avoiding selection bias to elucidate a more complete picture of the clinical characteristics, medications and outcomes of patients with FH. In this study, the primary endpoint is new ASCVD events. The secondary endpoints are as follows: LDL cholesterol, secondary ASCVD events and the occurrence of other diseases such as hypertension, diabetes or malignancies. It has been shown that patients with $\mathrm{FH}$ are at extremely high risk for the development of ASCVD, based on their lifelong elevation of LDL cholesterol. ${ }^{15}$ Recently, we have shown that the presence of FH-mutation is associated with higher risk for ASCVD. ${ }^{16}$ In addition, we have also shown that other traditional risk factors, such as hypertension and diabetes, also exacerbate their phenotype. ${ }^{17}$ Accordingly, we are assessing such factors comprehensively in this study to further determine the impact of those factors among the patients. In addition, screening for subclinical atherosclerosis using carotid ultrasound and/or CT for FH on a regular basis (every 2 to 3 years) is recommended in our clinical practice. In this way, we believe that we can assess the development of subclinical as well as clinical atherosclerosis in FH in this study. Moreover, there are no exclusion criteria regarding the means of referral to our hospitals. We anticipate many patients with FH identified through cascade screening will be included in this study. In this way, the inclusion of patients from different age groups, and especially younger patients, will help us estimate the starting age of the development of atherosclerosis in patients with $\mathrm{FH}$.

Our intention to perform comprehensive genetic testing for all patients is a strength of this study. This will both enable us to confirm diagnosis of $\mathrm{FH}$ and future ASCVD risk, and genetic testing will also facilitate decision-making during treatment and promote cascade screening among the patients' relatives. In addition, data from this will help us improve the present FH clinical 
diagnostic criteria in Japan and potentially across the world. In this study, genetic analyses will be performed as a part of the research and the results will be returned to the patients, with genetic counselling available on request. We are also now conducting another clinical trial (Impact of Genetic Testing on LDL Cholesterol in Patients with FH (GenTLe-FH)), which aims to assess the clinical benefits of genetic testing in FH subjects. ${ }^{18}$ Our studies may provide interesting perspectives in returning genetic results with the disclosure of future ASCVD risk for patients with $\mathrm{FH}$, and providing them with reliable and evidence-based treatment options to reduce their risk. We may associate our initiative in time with another Japanese FH registry, the PROLIPID-FH registry. By doing so, our shared data should be more representative of the entire country.

This study has several limitations. First, patients are not involved in the design of this study, since contacting patients for research purpose raises challenges in Japan. Involvement in the design of the registry may have otherwise provided us with useful information. Second, there will be no interaction between the registry information and physicians until the initial report is published officially. Part of the reason for this is we wanted to observe the current status/situations of FH treatments and prognoses in Japan in an unbiassed manner. However, we will be providing useful feedback from this registry not only to the physicians on charge of patient care, but also to interested observers across the world through scientific papers once the determined study period is over. Finally, it is unfortunate that we cannot collect information about dietary habits and physical activity in this study. However, we will be collecting data concerning changes in body weight and waist circumference. Accordingly, we hope to be able to estimate the effects of lifestyle in patients with FH.

In conclusion, we aim to demonstrate the study design and protocol of the Hokuriku-plus FH registry. We hypothesise that patients with FH-mutation and/or other traditional risk factors are associated with a higher risk of ASCVD, and that this higher risk can be cancelled out through earlier and/or more intensive LDL-cholesterollowering therapies. We expect to see that a portion of the clinical-FH patients have sitosterolemia and/or are carriers of the $A B C G 5$ or $A B C G 8$ gene mutation who have different aetiology as well as different prognosis from true $\mathrm{FH} .{ }^{19}$ Finally, this study will provide insights into the importance of clinical and genetic diagnosis of $\mathrm{FH}$ with extremely high cardiovascular risk.

\footnotetext{
Author affiliations

${ }^{1}$ Department of Cardiovascular Medicine, Graduate School of Medical Sciences, Kanazawa University, Kanazawa, Japan

${ }^{2}$ Department of Laboratory Science, Molecular Biochemistry and Molecular Biology, Graduate School of Medical Science, Kanazawa University, Kanazawa, Japan ${ }^{3}$ Innovative Clinical Research Center, Kanazawa University, Kanazawa, Japan ${ }^{4}$ Department of Cardiology, Ishikawa Prefectural Central Hospital, Kanazawa, Japan ${ }^{5}$ Department of Cardiology, Kanazawa Cardiovascular Hospital, Kanazawa, Japan

${ }^{6}$ Division of Internal Medicine, Saiseikai Kanazawa Hospital, Kanazawa, Japan
}

${ }^{7}$ Department of Internal Medicine, JCHO Kanazawa Hospital, Kanazawa, Japan ${ }^{8}$ Department of Internal Medicine, KKR Hokuriku Hospital, Kanazawa, Japan ${ }^{9}$ Department of Internal Medicine, Kanazawa-Nishi Hospital, Kanazawa, Japan

${ }^{10}$ Department of Internal Medicine, Hokuriku Central Hospital of Japan Mutual Aid Association of Public School Teachers, Oyabe, Japan

${ }^{11}$ Department of Internal Medicine, Suzu City General Hospital, Suzu, Japan

${ }^{12}$ Department of Internal Medicine, Wajima City Hospital, Wajima, Japan

${ }^{13}$ Department of Cardiology, Keiju General Hospital, Nanao, Japan

${ }^{14}$ Department of Cardiology, Houju Memorial Hospital, Nomi, Japan

${ }^{15}$ Department of Internal Medicine, Komatsu Municipal Hospital, Komatsu, Japan

${ }^{16}$ Department of Internal Medicine, Kaga Medical Center, Kaga, Japan

${ }^{17}$ Department of Cardiology, Fukui Prefectural Hospital, Fukui, Japan

${ }^{18}$ Department of Cardiology, Fukui CardioVascular Center, Fukui, Japan

${ }^{19}$ Department of Cardiology, Toyama Red Cross Hospital, Toyama, Japan

${ }^{20}$ Department of Cardiology, Koseiren Takaoka Hospital, Takaoka, Japan

${ }^{21}$ Department of Cardiology, Takaoka City Hospital, Takaoka, Japan

${ }^{22}$ Department of Cardiology, Yokohama Sakae Kyosai Hospital, Yokohama, Japan

${ }^{23}$ Division of Cardiology, Department of Internal Medicine, Toyama Prefectural

Central Hospital, Toyama, Japan

${ }^{24}$ Department of Cardiology, Toyama City Hospital, Toyama, Japan

${ }^{25}$ Department of Cardiology, Kurobe City Hospital, Kurobe, Japan

${ }^{26}$ Department of Cardiology, Tonami General Hospital, Tonami, Japan

${ }^{27}$ Department of Cardiology, Public Central Hospital of Matto Ishikawa, Hakusan, Japan

${ }^{28}$ Department of Cardiology, Kanazawa City Hospital, Kanazawa, Japan

${ }^{29}$ Department of Cardiology, Kanazawa Arimatsu Hospital, Kanazawa, Japan

${ }^{30}$ Department of Cardiology, Kanazawa Red Cross Hospital, Kanazawa, Japan

${ }^{31}$ Department of Cardiology, National Hospital Organization Kanazawa Medical

Center, Kanazawa, Japan

${ }^{32}$ Department of Cardiology, Yawata Medical Center, Komatsu, Japan

${ }^{33}$ Department of Internal Medicine, Hakui Public Hospital, Hakui, Japan

${ }^{34}$ Department of Internal Medicine, Noto General Hospital, Nanao, Japan

${ }^{35}$ Department of Internal Medicine, Fukui-ken Saiseikai Hospital, Fukui, Japan

${ }^{36}$ Department of Cardiology, Municipal Tsuruga Hospital, Tsuruga, Japan

${ }^{37}$ Department of Internal Medicine, Tsurugi Hospital, Hakusan, Japan

${ }^{38}$ Department of Cardiology, Kouseiren Namerikawa Hospital, Namerikawa, Japan

${ }^{39}$ Division of Cardiology, Kanazawa Medical University Hospital, Kahoku-gun, Japan

Twitter Akihiro Nomura @nomuryn29

Acknowledgements We express our gratitude to all the participants and staff in this study.

Contributors All of the authors (HTa, HOK, SY, MS, ANom, TT, MMo, S-iT, TKato, SU, KS, KH, NF, Aln, STakah, Ylm, TMat, ANoh, KeM, MNam, HTe, TY, TAr, MMi, TAb, YIt, CN, SKaw, YT, JK, YK, HMa, HS, AH, HI, TH, MuT, KaM, MY, YN, KOs, TMab, TI, BK, SKat, MF, KUc, KF, TNa, TNo, IM, KUs, KOt, KOk, SHi, IA, KKu, OT, SHo, HOd, STakata, $\mathrm{HMu}, \mathrm{MK}, \mathrm{HN}, \mathrm{YS}$, SS, WO, CF, TKats, HOo, Alg, MNak, SO, KoM, YM, NS, MY, HA, $\mathrm{KKu}, \mathrm{MaT}$ and $\mathrm{M}-\mathrm{aK}$ ) designed, conducted the study, performed a critical review and approved the final version of this manuscript. STakah and YIm gave us critical comments on statistical methods.

Funding This study is supported by the Ministry of Health, Labor and Welfare Research Grant for Research on Rare and Intractable Diseases, and Japanese Circulation Society (project for genome analysis in cardiovascular diseases).

Map disclaimer The depiction of boundaries on this map does not imply the expression of any opinion whatsoever on the part of BMJ (or any member of its group) concerning the legal status of any country, territory, jurisdiction or area or of its authorities. This map is provided without any warranty of any kind, either express or implied.

Competing interests None declared.

Patient and public involvement Patients and/or the public were not involved in the design, or conduct, or reporting or dissemination plans of this research.

Patient consent for publication Not required.

Provenance and peer review Not commissioned; externally peer reviewed.

Data availability statement Data are available upon reasonable request. Data including detailed study protocol will be available upon official request to $\mathrm{Dr}$, Hayato Tada (Email: ht240z@med.kanazawa-u.ac.jp). 
Open access This is an open access article distributed in accordance with the Creative Commons Attribution Non Commercial (CC BY-NC 4.0) license, which permits others to distribute, remix, adapt, build upon this work non-commercially, and license their derivative works on different terms, provided the original work is properly cited, appropriate credit is given, any changes made indicated, and the use is non-commercial. See: http://creativecommons.org/licenses/by-nc/4.0/.

\section{ORCID iDs}

Hayato Tada http://orcid.org/0000-0002-3357-1809

Akihiro Nomura http://orcid.org/0000-0001-6647-8240

\section{REFERENCES}

1 Harada-Shiba M, Arai H, Ishigaki Y, et al. Guidelines for diagnosis and treatment of familial hypercholesterolemia 2017. J Atheroscler Thromb 2018;25:751-70.

2 Gidding SS, Champagne MA, de Ferranti SD, et al. The agenda for familial hypercholesterolemia: a scientific statement from the American heart association. Circulation 2015:132:2167-92.

3 Watts GF, Gidding S, Wierzbicki AS, et al. Integrated guidance on the care of familial hypercholesterolaemia from the International $\mathrm{FH}$ Foundation. Int J Cardiol 2014:171:309-25.

4 Nordestgaard BG, Chapman MJ, Humphries SE, et al. Familial hypercholesterolaemia is underdiagnosed and undertreated in the general population: guidance for clinicians to prevent coronary heart disease: consensus statement of the European atherosclerosis Society. Eur Heart J 2013;34:3478-90.

5 Mabuchi H, Nohara A, Noguchi T, et al. Molecular genetic epidemiology of homozygous familial hypercholesterolemia in the Hokuriku district of Japan. Atherosclerosis 2011;214:404-7.

6 Mabuchi $\mathrm{H}$. Half a century tales of familial hypercholesterolemia $(\mathrm{FH})$ in Japan. J Atheroscler Thromb 2017;24:189-207.

7 Versmissen J, Oosterveer DM, Yazdanpanah M, et al. Efficacy of statins in familial hypercholesterolaemia: a long term cohort study. BMJ 2008;337:a2423.

8 Harada-Shiba M, Sugisawa T, Makino H, et al. Impact of statin treatment on the clinical fate of heterozygous familial hypercholesterolemia. J Atheroscler Thromb 2010;17:667-74.
9 van der Graaf A, Cuffie-Jackson C, Vissers MN, et al. Efficacy and safety of coadministration of ezetimibe and simvastatin in adolescents with heterozygous familial hypercholesterolemia. J Am Coll Cardiol 2008;52:1421-9.

10 Neil HAW, Huxley RR, Hawkins MM, et al. Comparison of the risk of fatal coronary heart disease in treated xanthomatous and non-xanthomatous heterozygous familial hypercholesterolaemia: a prospective registry study. Atherosclerosis 2003;170:73-8.

11 O'Brien EC, Roe MT, Fraulo ES, et al. Rationale and design of the familial hypercholesterolemia Foundation cascade screening for awareness and detection of familial hypercholesterolemia registry. Am Heart J 2014;167:342-9.

12 Mata N, Alonso R, Badimón L, et al. Clinical characteristics and evaluation of LDL-cholesterol treatment of the Spanish familial hypercholesterolemia longitudinal cohort study (SAFEHEART). Lipids Health Dis 2011;10:94.

13 Tada H, Kawashiri M-A, Nomura A, et al. Oligogenic familial hypercholesterolemia, LDL cholesterol, and coronary artery disease. $J$ Clin Lipidol 2018;12:1436-44.

14 Harris PA, Taylor R, Thielke R, et al. Research electronic data capture (REDCap)--a metadata-driven methodology and workflow process for providing translational research informatics support. J Biomed Inform 2009;42:377-81.

15 Nordestgaard BG, Chapman MJ, Humphries SE, et al. Familial hypercholesterolaemia is underdiagnosed and undertreated in the general population: guidance for clinicians to prevent coronary heart disease: consensus statement of the European atherosclerosis Society. Eur Heart J 2013;34:3478-90.

16 Tada H, Kawashiri M-A, Nohara A, et al. Impact of clinical signs and genetic diagnosis of familial hypercholesterolaemia on the prevalence of coronary artery disease in patients with severe hypercholesterolaemia. Eur Heart J 2017;38:1573-9.

17 Tada H, Kawashiri M-A, Nohara A, et al. Assessment of arterial stiffness in patients with familial hypercholesterolemia. $J$ Clin Lipidol 2018;12:397-402.

18 Nomura A, Tada H, Okada $\mathrm{H}$, et al. Impact of genetic testing on low-density lipoprotein cholesterol in patients with familial hypercholesterolemia (GenTLe-FH): a randomised waiting list controlled open-label study protocol. BMJ Open 2018;8:e023636.

19 Tada H, Okada H, Nomura A, et al. Rare and deleterious mutations in ABCG5/ABCG8 genes contribute to mimicking and worsening of familial hypercholesterolemia phenotype. Circ J 2019;83:1917-24. 